\title{
Harmonic Wideband simultaneous dual-frequency MR Elastography
}

$\underline{\text { Pilar Sango Solanas }}{ }^{1}$, Kevin Tse Ve Koon ${ }^{1}$, Helene Ratiney ${ }^{1}$, Fabien Millioz ${ }^{1}$, Cyrielle Caussy $^{2,3}$ and Olivier Beuf ${ }^{*}$

1 Univ Lyon, INSA-Lyon, Université Claude Bernard Lyon 1, UJM-Saint Etienne, CNRS, Inserm, CREATIS UMR 5220, U1206, F-69616, Lyon, France

${ }^{2}$ Univ Lyon, CarMen Laboratory, INSERM, INRA, INSA Lyon, Université Claude Bernard Lyon 1,69495 Pierre-Bénite, France

${ }^{3}$ Hospices Civils de Lyon, Département Endocrinologie, Diabète et Nutrition, Hôpital Lyon Sud, 69495 Pierre-Bénite, France

Word count: 4407

Key words (3): MR Elastography, multifrequency, phase offset undersampling

Corresponding author:

Olivier BEUF, PhD

CREATIS CNRS UMR 5220

INSA Lyon, Bât Blaise Pascal (502)

7 Avenue Jean Capelle

F-69621 VILLEURBANNE

Tél. +33472438520

olivier.beuf@creatis.insa-lyon.fr 


\section{List of abbreviations}

$A_{\text {exc }}$, excitation shear wave amplitude

$f_{M E G}$, motion encoding gradient oscillating frequency

$f_{\text {exc }}$, excitation shear wave frequency

$f_{S}$, sampling frequency

G', shear storage modulus

G", shear loss modulus

$\mathrm{G}^{*}$, complex shear modulus

MEG, motion encoding gradient

MRE, Magnetic Resonance Elastography

NAFLD, Nonalcoholic fatty liver disease

$\mathrm{N}_{\mathrm{G}}$, number of motion encoding gradient cycles

PNR, Phase-to-Noise Ratio

$q$, fractional encoding ratio

RARE, Rapid Acquisition with Refocused Echoes

ROI, Region of interest

SNR, Signal-to-Noise Ratio. 


\section{SUMMARY ABSTRACT}

Magnetic resonance elastography (MRE) is used to non-invasively quantify viscoelastic properties of tissues based on the measurement of propagation characteristics of shear waves. Because some of these viscoelastic parameters show a frequency dependence, multifrequency analysis allows us to measure the wave propagation dispersion, leading to a better characterization of tissue properties. Conventionally, motion encoding gradients (MEGs) oscillating at the same frequency as the mechanical excitation encode motion. Hence, multifrequency data is usually obtained by sequentially repeating monochromatic wave excitations experiments at different frequencies. The result is that the total acquisition time is multiplied by a factor corresponding to the number of repetitions of monofrequency experiments, which is a major limitation of multifrequency MRE. In order to make it more accessible, a novel single-shot harmonic wideband dualfrequency MRE method is proposed. Two superposed shear waves of different frequencies are simultaneously generated and propagate in a sample. Trapezoidal oscillating MEGs are used to encode mechanical vibrations having frequencies that are an odd multiple of the MEG frequency. The number of phase offsets is optimized to reduce the acquisition time. For this purpose, a sampling method not respecting the Shannon theorem is used to produce a controlled temporal aliasing that allows us to encode both frequencies without any additional examination time. Phantom experiments were run to compare conventional monofrequency MRE with the single-shot dual-frequency MRE method and showed excellent agreement between the reconstructed shear storage moduli $G^{\prime}$. In addition, dual-frequency MRE yielded an increased signal-to-noise ratio compared with conventional monofrequency MRE acquisitions when encoding the high frequency component. The novel proposed multifrequency MRE method could be applied to simultaneously acquire more than two frequency components, reducing examination time. Further studies are needed to confirm its applicability in preclinical and clinical models. 


\section{INTRODUCTION}

The development of a disease is associated with morphological and structural changes in the affected organs. Various imaging techniques enable to obtain images of organs as well as the non-invasive assessment of their different physical characteristics. Mechanical properties of these tissues are of particular interest as they undergo appreciable modifications which can be quantified ${ }^{1}$, enabling evaluation of the stage of a disease or assessment of longitudinal changes such as progression or regression of a disease stage. Elastography techniques using ultrasound ${ }^{2}$ or $\mathrm{MRI}^{3}$ are used to non-invasively assess the stiffness of tissues by providing information on viscoelastic properties. Specifically, magnetic resonance elastography 3 (MRE) quantifies, based on viscoelastic models, the mechanical properties of tissues from measurements of the shear wave propagation through an organ.

Several studies have already shown that MRE is a relevant non-invasive tool in clinical practice for characterizing liver fibrosis disease evolution by providing comparable results to liver biopsy ${ }^{4-7}$ without being limited to a small number of samples. Actually, MRE is a recognized non-invasive modality to assess liver fibrosis stage in patients with nonalcoholic fatty liver disease (NAFLD). Indeed, MRE generally outperforms ultrasound modalities for the staging of liver fibrosis in patients with NAFLD ${ }^{6,8}$. Clinical researches have also exploited the valuable tissue mechanical characterization technique of MRE to target other organs with different applications ${ }^{9}$ such as the diagnosis of cardiac abnormalities $^{10-12}$, breast cancer ${ }^{13-16}$ and brain viscoelastic properties measurement ${ }^{17-}$ 20. However, the utility of MRE in clinical practice is limited by its long acquisition duration 21 .

The MRE biomarker currently used to assess the severity of the different mentioned diseases is the shear storage modulus $\left(G^{\prime}\right)$ or more rarely the complex shear modulus $\left(G^{*}\right)$, both parameters are frequency dependent and represent elastic and viscous characteristics of tissues. The frequency dispersion of G' over a wide dynamic range can be evaluated to detect microstructural ${ }^{22-24}$ changes, providing thus a more detailed mechanical characterization of tissues ${ }^{22,25}$. It was demonstrated that viscoelastic powerlaw behavior of living soft tissue is altered by disease 26,27 and presently, multifrequency MRE is one of the few imaging-based techniques able to measure the dispersion of the complex shear modulus of soft tissue in vivo. 
In conventional MRE, oscillating motion encoding gradients (MEGs) encode the cyclic motion of nuclear spins when both MEGs and spins are oscillating at the same frequency ${ }^{3,21}$. But there are also other motion encoding strategies in which oscillating MEGs are not needed $28-32$.

Conventionally, to encode low excitation frequencies, long MEGs are used. This implies an increase in the TE and consequently, the signal to noise ratio (SNR) is degraded especially for short T2 tissues. High excitation frequencies can overcome this limitation. However, they are not appropriate for soft deep tissues for which shear waves are quickly attenuated. Each acquisition provides the mechanical information of a single excitation frequency and multifrequency results have to be obtained by repeating the experiences with monochromatic wave excitations at different frequencies. This yields very long total acquisition times, which is one of the major limitations of the technique in routine clinical practice. Therefore, reducing its duration would ultimately improve its efficiency, reduce its financial cost and thus increase its availability in the future.

In the last decades some multifrequency MRE methods have been developed. To this aim, the resulting spectral sensitivity of a time-limited sinusoidal MEGs has been exploited: for a given MEG, motion of spins oscillating at a frequency contained in the MEG's sensitivity bandwidth can be encoded.

The study by Rump et al. ${ }^{33}$ initially proposed a fractional encoding MRE method in which only a fraction of one vibration cycle per TR is used for motion sensitization, defined by the fractional encoding ratio $\left(q=\frac{f_{e x c}}{f_{M E G}}\right)$ where $f_{M E G}$ and $f_{e x c}$ are the frequencies of the MEG and the mechanical excitation respectively. In the liver, they used a $100 \mathrm{~Hz}$ MEG to encode a $76 \mathrm{~Hz}$ excitation frequency $(q=0.76)$. This approach demonstrated that low frequencies can be encoded with shorter TEs yielding higher SNR. From then on, several studies have exploited this strategy for different applications ${ }^{10,26,34-37}$. For instance, Klatt et al. $^{34}$ and Asbach et al. ${ }^{35}$ demonstrated the high consistency and reproducibility of MRE in human liver when using a $50 \mathrm{~Hz}$ sinusoidal MEG to simultaneously encode four superimposed sinusoidal waveforms with frequencies of $25.0,37.5,50.0$, and $62.5 \mathrm{~Hz}$ $(q=0.5,0.75,1,1.25)$. And more recently, Garteiser et al. ${ }^{37}$ introduced a MRE sequence that enabled the acquisition of multiple frequencies on several slices in the same examination. These approaches proved the feasibility of fractional encoding to simultaneous acquire several mechanical excitation frequencies to extend the dynamic range of a single MRE 
examination instead of being monochromatic and therefore increasing the acquisition speed.

Despite these advantages, fractional encoding is usually limited in vivo to relatively large targeted organs where low frequencies are needed. Current methods are thus not suitable for the encoding of motion in small organs where higher frequencies are required. In view of carrying out multifrequency MRE and extracting the subsequent power laws, accessing data covering much higher frequency ranges would represent a substantial added value. However, this strategy is confronted to two major limitations. The first limitation, as previously mentioned, is linked to the higher attenuation of shear waves at higher frequencies, which therefore limits their penetration depth and the region of interest. The second limitation is related to hardware specifications of MR scanners. Indeed, gradient amplitudes and switching rates are limited which is particularly true in clinical systems. Thus, even if we were able to generate and propagate higher frequencies shear waves, encoding them in the phase of the MR signal would be challenging.

Here, we first propose a harmonic wideband MRE method, demonstrated by applying low oscillating MEG frequencies to encode higher frequencies, usually hard to be reached by the gradient system of MRI scanners. In this way, we overcome the limitation due to the hardware of MR scanner although a remaining challenge is the propagation of high frequencies through tissues. Then, this strategy is combined with a dual-frequency mechanical excitation, yielding the simultaneous encoding and acquisition of both frequency motions. This allows exploring a wider range of frequencies that may improve the performance of the power-law fit analysis of viscoelastic parameters frequency dispersion. Finally, the number of phase offset is optimized in order to still reduce the total acquisition time. For this purpose, a non-respecting Shannon sampling method is used to produce a controlled temporal aliasing that allows to encode both frequencies in a minimal acquisition time.

The main objectives of this study were first, to demonstrate the feasibility of the novel single-shot wideband dual-frequency method in phantom experiments and second, to test the phase offset undersampling method based on temporal aliasing, allowing to significantly reduce the total acquisition time. 


\section{THEORETICAL BACKGROUND}

\section{Motion encoding and viscoelastic parameters reconstruction}

MRE is a phase-contrast motion encoding method. An oscillating gradient $\overrightarrow{\boldsymbol{G}}(t)$ encodes the cyclic motion of a spin $\vec{r}(t)$ at a given frequency into the MRI signal phase $\phi^{3}$ :

$$
\phi=\gamma \int_{t_{0}}^{t_{0}+T_{e n c}} \overrightarrow{\boldsymbol{G}}(t) \cdot \vec{r}(t) d t
$$

where $\gamma$ is the gyromagnetic ratio and $T_{\text {enc }}$ denotes the application time of MEG cycles.

The cumulated phase reaches maximum values when the frequency of motion is equal to the MEG frequency. However even for sinusoidal MEGs, this cumulated phase is still appreciable if the motion frequency belongs to the sensitivity band of the MEG as shown in Figure 1a. This is the underlying principle of fractional encoding. This sensitivity band has a periodical behavior when using a trapezoidal-shaped MEG since the latter can be decomposed as a Fourier series that is as a sum of odd-harmonics: non-resonant sensitivity bands appear around the odd-multiples of the MEG frequency $\left(f_{\text {exc }}=\right.$ $(2 n+1) \cdot f_{M E G}$ with $\left.n=0,1,2 \ldots\right)$ as can be seen in Figure $1 b$.

Equation [1] was used prior to the experiments to numerically calculate the cumulated encoded phase at the driving frequency used. We considered a shear excitation frequency

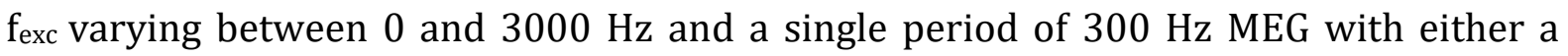
sinusoidal (Figure 1a) or a trapezoidal shape (Figure 1b). Here, we can see the sensitivity bands around 300, 900, 1500 and $2100 \mathrm{~Hz}$ that appear when using a $300 \mathrm{~Hz}$ trapezoidal MEG.

In our experiments, we considered only plane waves and only one direction was sensitized with the MEGs. The propagating wave was represented by a scalar wave field $\mathrm{u}(\mathrm{x}, \mathrm{y}, \mathrm{t})$ that was proportional to the unwrapped accumulated phase $\left(\varphi_{\text {unw }}\right)$. The displacement in each pixel was calculated as follow:

$$
u(x, y, t)=C \cdot \varphi_{u n w}(x, y, t),
$$

where $C$ is a conversion factor depending on the MEGs amplitude, the excitation wave frequency and amplitude. The number of acquired phase offsets gave the size of the temporal dimension. 
Afterwards, a temporal Fourier transform was applied to the wave field in order to work in the frequency domain $\mathrm{U}(\mathrm{x}, \mathrm{y}, \omega)$. In the case of dual-frequency acquisitions, the two harmonics corresponding to both excitation frequencies are kept.

The Helmholtz equation inversion applied to a spin harmonically oscillating at a given frequency is expressed by

$$
\rho \omega^{2} U(x, y, \omega)+G^{*}(\omega) \Delta U(x, y, \omega)=0,
$$

where $G^{*}(\omega)$ and $\rho$ denote the complex shear modulus and the density of the tissue respectively. From this equation we can derive the expression of $G^{*}(\omega)$.

The complex shear modulus can be expressed by $G^{*}=G^{\prime}+i G^{\prime \prime}$, where $G^{\prime}$ represents the storage modulus, which is related to the tissue elasticity and G" the loss modulus, related to the viscosity of the medium.

In our results, we focused on the value of the parameter $G^{\prime}$ but G" were also calculated. Values for different excitation frequencies and with different acquisition modes (mono and dual-frequency acquisitions) are compared.

\section{Undersampling}

According to the Nyquist-Shannon sampling theorem, $f_{s}>2 \cdot f_{\max }$, where $f_{s}$ is the sampling frequency and $f_{\text {max }}$ is the maximum frequency contained in the acquired signal.

For a correct sampling of a monochromatic wave, at least three sampling steps equally spaced over a period of the signal wave are needed. But in fact, in conventional MRE, four equally spaced phase offsets are usually acquired in order to assure the acquisitions of maxima and minima phase points in the shear wave time evolution. This implies that to do repetitive monofrequency acquisitions at $n$ different mechanical excitation frequencies, the total acquisition time would ben times greater.

However, in our specific case of a two components wave motion with an odd harmonic relationship between them $\left(f_{2}=3 f_{1}\right)$, a small number of phase offsets could be able to encode both frequencies because of the temporal aliasing that appears when the Shannon theorem is not respected to sample polychromatic waves. This aliasing phenomenon is shown in Figure 2. Here, we considered a polychromatic wave resulting from the summation of two shear waves of $f_{1}=300 \mathrm{~Hz}$ and $f_{2}=900 \mathrm{~Hz}$ and we realized a sampling 
with a sampling period $\mathrm{T}_{\mathrm{s}}=\mathrm{T}_{1} / \mathrm{N}=1 /\left(\mathrm{N}_{1}\right)$ (this means that we acquire $\mathrm{N}$ equally-spaced time points over one period of the fundamental frequency shear wave). As the spectrum of the original signal (represented as a block in dashed lines) is replicated every $\mathrm{f}_{\mathrm{s}}$, when the sampling frequency respects the Nyquist criterion ( $\mathrm{N}$ should be greater than 6 since the second component $\mathrm{f}_{2}=3 \mathrm{f}_{1}$ ), there is no overlap between adjacent blocks (Figure $2 \mathrm{a}$ ). When the Nyquist criterion is not respected, the blocks overlap leading to contamination from components of adjacent blocks (Figure 2b-c). For instance, in Figure 2b, both frequencies overlap in the same acquired points meaning the two components cannot be separated. However, as can be seen in Figure 2c, due to the fact that the second sought component is an odd harmonic of the first one, even in the case of 5 sampling steps, the resulting aliasing doesn't prevent separation of the two components since the aliased higher component corresponds to a different reconstructed point than the lower component in Fourier space. Therefore, such a chosen configuration with odd harmonics in addition to a fundamental component produces a controlled frequency aliasing meaning that it is possible to encode simultaneously several odd multiples of the fundamental frequency with a smaller number of phase offsets. In general, to simultaneously encode $n$ different shear wave frequencies, $2 n+1$ phase offsets would be needed.

\section{MATERIALS AND METHODS}

\section{MRE setup}

All acquisitions were performed on a preclinical 4.7 T MRI scanner (BioSpec Bruker system, Ettlingen, Germany), with a $60 \mathrm{~mm}$ inner diameter volume coil in transmit/receive mode.

Experiments were carried out on a uniform phantom composed of $75 \%$-standard plastisol $^{38}$ (Plastileurre Standard, Bricoleurre, France) and 25\% softener (Di-2ethylhexyl adipate). Relaxation times measured using a RARE sequence with different TEs and TRs gave $[\mathrm{T} 1, \mathrm{~T} 2]=[300,35] \mathrm{ms}$. Although the phantom used here could not be characterized from a mechanical point of view by a complementary method, in previous studies we compared values of storage and loss moduli obtained both with MRE and 
High-Frequency Rheometry for different plastisol phantoms, confirming that the complex modulus of plastisol phantoms can be reliably assessed using $\mathrm{MRE}^{38}$.

A piezoelectric actuator (CEDRAT Technologies) was used as mechanical transducer for motion generation. A dual output wave generator was used to produce two harmonic signals of frequencies 300 and $900 \mathrm{~Hz}$. They were then summed and fed to the actuator thus resulting in the latter generating waves at both frequencies.

\section{MRE acquisition}

The MRE sequence used was based on a conventional RARE sequence. Inserted MEGs may have variable sinusoidal or trapezoidal shapes with adjustable frequencies and amplitudes. Two acquisitions with inversion of the MEG polarity were made for phase images subtraction to reduce static phase errors. Oscillating MEGs were synchronized with the mechanical excitation. The wave generator was triggered the beginning of each TR in order to reach a steady-state wave propagation during the MEGs application period.

Fixed acquisition parameters were axial orientation, TE/TR $=16.67 / 1000 \mathrm{~ms}$, single slice of $1 \mathrm{~mm}$ thickness, field of view $=4 \times 4 \mathrm{~cm}^{2}$, matrix size $=128 \times 128$, RARE factor $=4$.

The number of MEG cycles $\left(\mathrm{N}_{\mathrm{G}}\right)$ and the amplitude of the shear waves (Aexc) were adjusted according to the excitation frequency. In order to account for the reduction of the encoding efficiency of the MEG at $900 \mathrm{~Hz}$, the amplitude of this shear wave component was tripled with respect to the $300 \mathrm{~Hz}$ component. The amplitude of MEGs was always the same $(250 \mathrm{mT} / \mathrm{m})$. A single direction was sensitized with the MEGs. Further details of the acquisitions are summarized in Table 1.

In our protocol, several acquisitions were realized: monochromatic acquisitions synchronized on the fundamental frequency with $f_{\text {exc }}=f_{M E G}$ (acquisitions 1 and 2), monochromatic acquisitions synchronized on the first odd harmonic with $f_{\text {exc }}=3 \cdot f_{\mathrm{MEG}}$ $(3,4)$ and a dual-frequency acquisition with $f_{M E G}=\min \left(f_{e x c}\right)(5)$. Numbers in brackets correspond to the acquisition numbers in Table 1 . During the dual-frequency acquisition (Figure 3), the oscillating trapezoidal gradients were fixed to the lowest frequency and allowed encoding of both frequencies due to the sensitive motion encoding wideband of MEGs, as explained previously in the MRE subchapter. According to the 
proposed phase offset undersampling method, five equally spaced phase offset of the dual-frequency acquisition were obtained.

To avoid artifacts during the viscoelastic parameter reconstruction, the lowest shear wave frequency was chosen so that the corresponding wavelength was smaller that the phantom dimension in the propagation direction. The pair of frequencies 300 and $900 \mathrm{~Hz}$ was considered in our work.

\section{Image analysis}

\section{Elastograms reconstruction}

Raw phase images were unwrapped with a quality guided path following phase unwrapping algorithm ${ }^{39}$. The proportional 2D displacement fields were temporally Fourier transformed. Then, each frequency was reconstructed from a different harmonic. In the multifrequency acquisition, the second and the fourth harmonic, which correspond to the fundamental frequency ( $\left.f_{1}\right)$ and to the high frequency $\left(f_{3}\right)$ respectively, were kept for the elastograms reconstruction.

Then, a spatial fourth-order 2D Butterworth filter with lower and upper thresholds of 0.25 and $2.5 \mathrm{~cm}^{-1}$ at $300 \mathrm{~Hz}$ and 1 and $3.5 \mathrm{~cm}^{-1}$ at $900 \mathrm{~Hz}$ was applied to filter and preserve the frequencies of interest as much as possible ${ }^{4,21}$. The spatial filter limits were chosen based on simulations of estimated wavelength values with different cut-offs filters values (data not shown).

Finally, the Helmholtz equation inversion ${ }^{40,41}$ (Equation 3) was applied to the displacement fields to calculate the shear storage modulus $G^{\prime}$ and the loss modulus G" at each frequency assuming a density of $1000 \mathrm{~kg} / \mathrm{m}^{3}$.

A 3x3 median filter was applied to the elastograms. A ROI (about 1600 pixels) was manually drawn in the center of the phantom, excluding the phantom borders in order to avoid reconstruction artifacts. The mean and standard deviation of G' and G' were calculated inside the ROI. The same ROI was used for all acquisitions and excitation frequencies.

\section{SNR and PNR analysis}

Signal-to-noise ratio (SNR) and Phase-to-Noise ratio (PNR) were extracted from magnitude and phase images respectively. SNR was calculated as the ratio of the mean 
signal of a ROI in the phantom to the standard deviation in the background. PNR was calculated as the product of the SNR and the phase encoding $\Delta(\operatorname{PNR}=\operatorname{SNR} \times \Delta)$. The phase encoding $\Delta$ is defined as the difference of the maximal and minimal phase encoded along a single line parallel to the wave propagation direction (Shown in Figure 4 at right). The PNR is calculated to evaluate the motion encoding efficiency of the different acquisition modes and image quality through SNR. TE and TR were always the same in order to acquire all scans under the same conditions. Note that all SNR were measured on the same regions of interest and the same phase offset of the wave propagation and line were taken for PNR computation so as to have a reliable estimation of the phase encoding.

\section{RESULTS}

The ability of the $300 \mathrm{~Hz}$ trapezoidal MEG to encode the $900 \mathrm{~Hz}$ excitation frequency was experimentally demonstrated as seen in Figure 4 and compared to the quasi-null encoded phase of a sinusoidal one.

The phase image of the dual-frequency excitation is showed in Figure 5, as well as phase images obtained from the inversion of the two separate frequency components after temporal Fourier transform. Both vibrations are correctly encoded. Likewise, the G' elastograms are computed for each excitation frequency. Both maps present some edge artifacts resulting from the viscoelastic parameters reconstruction method but they are otherwise quite uniform and conform to the homogeneous composition of the phantom.

The different acquisition modes are compared in terms of SNR, $\Delta$ and PNR in Figure 6. Only the acquisitions with trapezoidal MEGs are considered here. Dual-frequency results present the highest values of SNR yielding the best values of PNR. PNRs were 74.3, 67.4, 77.0, 82.7 rad (indicated respectively for acquisitions 1-3 and 5). Especially, we find an improved motion encoding efficiency of the highest frequency when using the $300 \mathrm{~Hz}$ MEG in both mono and dual-frequency acquisitions, compared to the conventional 900 Hz MEG monofrequency acquisition.

The mean values and standard deviation of G' obtained for both excitations frequencies by using the different acquisition modes are presented in Figure 7. Storage modulus values were $20.2 \pm 2,20.3 \pm 2.2 \mathrm{kPa}$ at $300 \mathrm{~Hz}$ (in acquisitions 1 and 5 respectively) and 
$23.6 \pm 3.7,23.9 \pm 2$ and $23.6 \pm 1.5 \mathrm{kPa}$ at $900 \mathrm{~Hz}$ (in acquisitions 2,3 and 5). Loss modulus values were $6.9 \pm 2.6,6.5 \pm 2.8 \mathrm{kPa}$ at $300 \mathrm{~Hz}$ (in acquisitions 1 and 5 respectively) and $8.3 \pm 1.6,7.9 \pm 3.8$ and $7.7 \pm 1.4 \mathrm{kPa}$ at $900 \mathrm{~Hz}$ (in acquisitions 2, 3 and 5). Results obtained were comparable between mono and dual-frequency acquisitions. The undersampling method shows also consistent results.

\section{DISCUSSION}

This study demonstrates the feasibility of the novel single-shot harmonic wideband dualfrequency MRE method. The simultaneous excitation of two distant frequencies (300-900 $\mathrm{Hz}$ ) shear waves combined to the sensitive harmonic wideband of trapezoidal motion encoding gradients enabled the mechanical characterization of tissues without any additional acquisition time. In addition, this study demonstrates that the proposed undersampling method, based on a controlled frequency aliasing, allows to reduce the examination time up to $38 \%$ of the time required in conventional MRE.

Although a better mechanical characterization of tissues can be obtained with multifrequency MRE, it is mostly limited by the long acquisition times required ${ }^{21}$ to repeat monofrequency acquisitions. To increase the speed of multifrequency MRE acquisitions, other investigators proposed different methods to do simultaneous multifrequency MRE. First approaches utilized the hypothesis that spectrally selective gradient waveforms could be designed in order to detect multiple harmonics of the spin motion simultaneously ${ }^{42}$. Manduca et al. ${ }^{43}$ exploited this property and found excellent agreements of shear stiffness results between mono and multifrequency acquisitions. Similarly to our study, Manduca et al. used two frequencies farther apart that may better determine dispersion effects. However, this method was restricted in terms of SNR due to a limitation of the gradient waveform amplitude. The major advantage of our proposed method is that SNR and PNR of dual-frequency acquisitions were not reduced and were even slightly higher than those found with conventional monofrequency MRE as shown in Figure 7.

Currently, the MRE technique commonly used to simultaneously encode several driving frequencies is the fractional encoding and is based on the sensitivity band of motion encoding gradients. It was introduced by Rump et $a l^{33}$ and it has been exploited in several 
studies $26,34,35,37$. These studies have applied the fractional encoding strategy in vivo to encode low frequency shear waves in large targeted organs (liver, brain). The key difference with our study rests on the fractional encoding ratios (between 0.3 and 1.25 versus 3 in our case) while reducing the examination time by using a non-classical sampling method.

In the present work, the harmonic wideband sensitivity of trapezoidal MEGs enables to encode high excitation frequencies ( $>500 \mathrm{~Hz}$ in preclinical experiments) with lower MEG frequencies. This is an interesting property since high oscillating frequencies are not obtainable by most gradient systems due to their low switching rate of the oscillating polarity, especially in clinical MRI scanners. Moreover, even in preclinical setups, fast oscillating gradients are more prone to inducing image imperfections due to hardware limitations. This could explain why in our results we found a lower SNR and PNR when encoding the $900 \mathrm{~Hz}$ wave with a $900 \mathrm{~Hz}$ MEG compared to the efficacy of the $300 \mathrm{~Hz}$ MEG (Figure 6). Likewise, this could have an effect in the viscoelastic parameters reconstruction and could explain the higher standard deviation obtained for this acquisition (Figure 7). One solution could be to reduce the amplitude of MEGs but this would lead to low cumulated phases encoding, which is a very frequent imposed compromise. The benefit of using high frequencies is that they enable a better power-law fit. Recent preclinical studies have investigated the effect of the considered range of frequencies on the power law fit. Riek et $a l^{44}$ found a good match between the fit with low and high frequency ranges on ex vivo experiments. However, Reiter et al. ${ }^{45}$ found an increasing correlation of elasticity with the degree of liver fibrosis when using high mechanical excitation frequencies. In this context, our method could be useful to fill the whole spectrum with few acquisitions in order to reduce the total acquisition time.

In addition, by accessing the information of two different vibrations, we have no penalty in the acquisition time since the proposed sampling method allows to drastically reduce the number of phase offsets to be acquired. Such undersampling, based on a controlled frequency aliasing, allows to simultaneously encode several odd harmonics of the fundamental frequency. Generally, in order to simultaneously encode $n$ different shear wave frequencies with odd harmonics relationship, the acquisition of $2 n+1$ phase offsets is required. This could be easily implemented to extend the dynamic of MRE to more than two frequencies in future experiments. Our proposed method presents a higher acceleration speed of multifrequency MRE acquisitions when compared with current 
existent methods. For instance, Garteiser et al. ${ }^{37}$ acquire 8 phase offsets to encode 3 different frequencies. Considering that usually 4 phase offset are acquired to encode a monofrequency shear wave, the gain ratio would be 33\%. On the other hand, with our proposed method, it would be possible to encode 3 different frequencies with 7 phase offsets so the gain would be $42 \%$. Therefore, the gain ratio of our proposed method is slightly better.

The challenging limitation of our study is the increased damping of shear waves at higher frequencies that results in limiting the penetration depth and the region of interest. In addition, we could not reach very high frequencies since the mechanical actuator in our experiment could not support frequencies higher than $1 \mathrm{kHz}$. Obtaining a plane wave pattern propagating through the sample was an important condition to respect in order to avoid possible artifacts in the viscoelastic parameter reconstruction.

The fast attenuation of high frequency shear waves through the tissues combined to the long TEs obtained with the low frequency MEGS, makes complicated the application of our method for the actual targeted organs with MRE (liver, brain) in a clinical context. Nevertheless, since our method enables to encode lower wavelengths ( $\sim 5 \mathrm{~mm}$ ), it might be useful in a clinical context for a novel MRE targeted organ: the characterization of thin superficial tissues such as the skin ${ }^{46}$. Also our proposed method could be interesting for stiff tissues such as the cartilage ${ }^{47,48}$ that require much higher vibration frequencies than soft tissues.

The current study is the proof of concept of a novel multifrequency MRE method in a preclinical context. The experiments were carried on a homogenous phantom to realize a first validation of the proposed method. Since we can obtain very different wavelengths with our dual-frequency method, it might be useful for heterogeneous phantoms in order to provide a better visualization of interfaces between softer and stiffer parts of the phantom ${ }^{49}$. However, the attenuation of the high frequency component would still be a limitation.

Further work is needed to explore the mentioned clinical and preclinical applications of the proposed method. 


\section{CONCLUSION}

In this study, we present an alternative technique to the standard simultaneous multifrequency motion encoding: using harmonic widebands of motion encoding gradients, it is possible to significantly increase the frequency of shear waves used in MRE without increasing the burden on gradient systems. In addition, the application of a nonrespecting Shannon sampling, which produces a controlled temporal aliasing, enables also a notable decrease in acquisition times of multifrequency MRE. Both combinations could ultimately be used to increase the speed of multifrequency MRE acquisitions and further work is needed to explore the clinical and preclinical applications of the proposed method.

\section{ACKNOWLEDGEMENTS}

This work was performed within the framework of the LABEX PRIMES of University of Lyon (ANR-11-LABX-0063), within the program "Investissements d'Avenir" (ANR-11IDEX-0007) operated by the French National Research Agency (ANR). MR experiments were acquired on the PILoT platform, member of the France Life Imaging infrastructure (ANR-11-INBS-0006). 


\section{REFERENCES}

1. Greenleaf JF, Fatemi M, Insana M. Selected Methods for Imaging Elastic Properties of Biological Tissues. Annu Rev Biomed Eng. 2003;5(1):57-78. doi:10.1146/annurev.bioeng.5.040202.121623

2. Dickinson RJ, Hill CR. Measurement of soft tissue motion using correlation between A-scans. Ultrasound Med Biol. 1982;8(3):263-271. doi:10.1016/03015629(82)90032-1

3. Muthupillai R, Lomas D, Rossman P, Greenleaf J, Manduca A, Ehman R. Magnetic resonance elastography by direct visualization of propagating acoustic strain waves. Science. 1995;269(5232):1854-1857. doi:10.1126/science.7569924

4. Yin M, Talwalkar JA, Glaser KJ, Manduca A, Grimm RC, Rossman PJ, Fidler JL, Ehman RL. Assessment of Hepatic Fibrosis With Magnetic Resonance Elastography. Clin Gastroenterol Hepatol. 2007;5(10):1207-1213.e2. doi:10.1016/j.cgh.2007.06.012

5. Rouvière O, Yin M, Dresner MA, Rossman PJ, Burgart LJ, Fidler JL, Ehman RL. MR Elastography of the Liver: Preliminary Results. Radiology. 2006;240(2):440-448. doi:10.1148/radiol.2402050606

6. Hsu C, Caussy C, Imajo K, Chen J, Singh S, Kaulback K, Le M-D, Hooker J, Tu X, Bettencourt R, Yin M, Sirlin CB, Ehman RL, Nakajima A, Loomba R. Magnetic Resonance vs Transient Elastography Analysis of Patients With Nonalcoholic Fatty Liver Disease: A Systematic Review and Pooled Analysis of Individual Participants. Clin Gastroenterol Hepatol. 2019;17(4):630-637.e8. doi:10.1016/j.cgh.2018.05.059

7. Huwart L, Sempoux C, Vicaut E, Salameh N, Annet L, Danse E, Peeters F, ter Beek LC, Rahier J, Sinkus R, Horsmans Y, Van Beers BE. Magnetic Resonance Elastography for the Noninvasive Staging of Liver Fibrosis. Gastroenterology. 2008;135(1):32-40. doi:10.1053/j.gastro.2008.03.076

8. Tapper EB, Loomba R. Noninvasive imaging biomarker assessment of liver fibrosis by elastography in NAFLD. Nat Rev Gastroenterol Hepatol. 2018;15(5):274-282. doi:10.1038/nrgastro.2018.10

9. Glaser KJ, Manduca A, Ehman RL. Review of MR elastography applications and recent developments. J Magn Reson Imaging. 2012;36(4):757-774. doi:10.1002/jmri.23597

10. Sack I, Rump J, Elgeti T, Samani A, Braun J. MR elastography of the human heart: Noninvasive assessment of myocardial elasticity changes by shear wave amplitude variations. Magn Reson Med. 2009;61(3):668-677. doi:10.1002/mrm.21878

11. Kolipaka A, Araoz PA, McGee KP, Manduca A, Ehman RL. Magnetic resonance elastography as a method for the assessment of effective myocardial stiffness throughout the cardiac cycle. Magn Reson Med. 2010;64(3):862-870. doi:10.1002/mrm.22467 
12. Elgeti T, Beling M, Hamm B, Braun J, Sack I. Cardiac Magnetic Resonance Elastography. Invest Radiol. 2010;45(12):6.

13. Sinkus R, Tanter M, Xydeas T, Catheline S, Bercoff J, Fink M. Viscoelastic shear properties of in vivo breast lesions measured by MR elastography. Magn Reson Imaging. 2005;23(2):159-165. doi:10.1016/j.mri.2004.11.060

14. Sinkus R, Lorenzen J, Schrader D, Lorenzen M, Dargatz M, Holz D. High-resolution tensor MR elastography for breast tumour detection. Phys Med Biol. 2000;45(6):1649-1664. doi:10.1088/0031-9155/45/6/317

15. Lorenzen J, Sinkus R, Lorenzen M, Dargatz M, Leussler C, Röschmann P, Adam G. MR elastography of the breast:preliminary clinical results. RöFo - Fortschritte Auf Dem Geb Röntgenstrahlen Bildgeb Verfahr. 2002;174(7):830-834. doi:10.1055/s-200232690

16. McKnight AL, Kugel JL, Rossman PJ, Manduca A, Hartmann LC, Ehman RL. MR Elastography of Breast Cancer: Preliminary Results. Am J Roentgenol. 2002;178(6):1411-1417. doi:10.2214/ajr.178.6.1781411

17. Kruse SA, Rose GH, Glaser KJ, Manduca A, Felmlee JP, Jack CR, Ehman RL. Magnetic resonance elastography of the brain. NeuroImage. 2008;39(1):231-237. doi:10.1016/j.neuroimage.2007.08.030

18. Green MA, Bilston LE, Sinkus R. In vivo brain viscoelastic properties measured by magnetic resonance elastography. NMR Biomed. 2008;21(7):755-764. doi:10.1002/nbm.1254

19. Sack I, Beierbach B, Hamhaber U, Klatt D, Braun J. Non-invasive measurement of brain viscoelasticity using magnetic resonance elastography. NMR Biomed. 2008;21(3):265-271. doi:10.1002/nbm.1189

20. Murphy MC, Huston J, Jack CR, Glaser KJ, Manduca A, Felmlee JP, Ehman RL. Decreased brain stiffness in Alzheimer's disease determined by magnetic resonance elastography. J Magn Reson Imaging. 2011;34(3):494-498. doi:10.1002/jmri.22707

21. Mariappan YK, Glaser KJ, Ehman RL. Magnetic resonance elastography: A review. Clin Anat. 2010;23(5):497-511. doi:10.1002/ca.21006

22. Guo J, Posnansky O, Hirsch S, Scheel M, Taupitz M, Braun J, Sack I. Fractal network dimension and viscoelastic powerlaw behavior: II. An experimental study of structure-mimicking phantoms by magnetic resonance elastography. Phys Med Biol. 2012;57(12):4041-4053. doi:10.1088/0031-9155/57/12/4041

23. Bigot M, Chauveau F, Amaz C, Sinkus R, Beuf O, Lambert SA. The apparent mechanical effect of isolated amyloid- $\beta$ and $\alpha$-synuclein aggregates revealed by multi-frequency MRE. NMR Biomed. 2020;33(1). doi:10.1002/nbm.4174

24. Jugé L, Petiet A, Lambert SA, Nicole P, Chatelin S, Vilgrain V, Van Beers BE, Bilston LE, Sinkus R. Microvasculature alters the dispersion properties of shear waves - a multi-frequency MR elastography study: Microvasculature Alters the Dispersion 
Properties of Shear Waves. NMR Biomed. 2015;28(12):1763-1771. doi:10.1002/nbm.3438

25. Posnansky O, Guo J, Hirsch S, Papazoglou S, Braun J, Sack I. Fractal network dimension and viscoelastic powerlaw behavior: I. A modeling approach based on a coarse-graining procedure combined with shear oscillatory rheometry. Phys Med Biol. 2012;57(12):4023-4040. doi:10.1088/0031-9155/57/12/4023

26. Asbach P, Klatt D, Schlosser B, Biermer M, Muche M, Rieger A, Loddenkemper C, Somasundaram R, Berg T, Hamm B, Braun J, Sack I. Viscoelasticity-based Staging of Hepatic Fibrosis with Multifrequency MR Elastography. Radiology. 2010;257(1):8086. doi:10.1148/radiol.10092489

27. Sack I, Jöhrens K, Würfel J, Braun J. Structure-sensitive elastography: on the viscoelastic powerlaw behavior of in vivo human tissue in health and disease. Soft Matter. 2013;9(24):5672. doi:10.1039/c3sm50552a

28. Lewa CJ. Magnetic Resonance Imaging in the Presence of Mechanical Waves. Spectrosc Lett. 1991;24(1):55-67. doi:10.1080/00387019108018124

29. Chenevert TL, Skovoroda AR, O'donnell M, Emelianov SY. Elasticity reconstructive imaging by means of stimulated echo MRI. Magn Reson Med. 1998;39(3):482-490. doi:10.1002/mrm.1910390319

30. Lefebvre PM, Van Reeth E, Ratiney H, Beuf O, Brusseau E, Lambert SA, Glaser SJ, Sugny D, Grenier D, Tse Ve Koon K. Active control of the spatial MRI phase distribution with optimal control theory. J Magn Reson. 2017;281:82-93. doi:10.1016/j.jmr.2017.05.008

31. Van Reeth E, Lefebvre PM, Ratiney H, Lambert SA, Tesch M, Brusseau E, Grenier D, Beuf O, Glaser SJ, Sugny D, Tse-Ve-Koon K. Constant gradient elastography with optimal control RF pulses. J Magn Reson. 2018;294:153-161. doi:10.1016/j.jmr.2018.07.013

32. Strasser J, Haindl MT, Stollberger R, Fazekas F, Ropele S. Magnetic resonance elastography of the human brain using a multiphase DENSE acquisition. Magn Reson Med.2019;81(6):3578-3587. doi:10.1002/mrm.27672

33. Rump J, Klatt D, Braun J, Warmuth C, Sack I. Fractional encoding of harmonic motions in MR elastography. Magn Reson Med. 2007;57(2):388-395. doi:10.1002/mrm.21152

34. Klatt D, Hamhaber U, Asbach P, Braun J, Sack I. Noninvasive assessment of the rheological behavior of human organs using multifrequency MR elastography: a study of brain and liver viscoelasticity. Phys Med Biol. 2007;52(24):7281-7294. doi:10.1088/0031-9155/52/24/006

35. Asbach P, Klatt D, Hamhaber U, Braun J, Somasundaram R, Hamm B, Sack I. Assessment of liver viscoelasticity using multifrequency MR elastography. Magn Reson Med. 2008;60(2):373-379. doi:10.1002/mrm.21636 
36. Robert B, Sinkus R, Gennisson J-L, Fink M. Application of DENSE-MR-elastography to the human heart. Magn Reson Med. 2009;62(5):1155-1163. doi:10.1002/mrm.22124

37. Garteiser P, Sahebjavaher RS, Ter Beek LC, Salcudean S, Vilgrain V, Van Beers BE, Sinkus R. Rapid acquisition of multifrequency, multislice and multidirectional MR elastography data with a fractionally encoded gradient echo sequence: MULTIFREQUENCY MRE WITH GRADIENT ECHOES. NMR Biomed. 2013;26(10):1326-1335. doi:10.1002/nbm.2958

38. Lefebvre PM, Koon KTV, Brusseau E, Nicolle S, Palieme J-F, Lambert SA, Grenier D. Comparison of viscoelastic property characterization of plastisol phantoms with magnetic resonance elastography and high-frequency rheometry. In: 2016 38th Annual International Conference of the IEEE Engineering in Medicine and Biology Society (EMBC). IEEE; 2016:1216-1219. doi:10.1109/EMBC.2016.7590924

39. Ghiglia DC, Pritt MD. Two-Dimensional Phase Unwrapping: Theory, Algorithms, and Software. Wiley; 1998.

40. Manduca A, Oliphant TE, Dresner MA, Mahowald JL, Kruse SA, Amromin E, Felmlee JP, Greenleaf JF, Ehman RL. Magnetic resonance elastography: Non-invasive mapping of tissue elasticity. Med Image Anal. 2001;5(4):237-254. doi:10.1016/S1361-8415(00)00039-6

41. Oliphant TE, Manduca A, Ehman RL, Greenleaf JF. Complex-valued stiffness reconstruction for magnetic resonance elastography by algebraic inversion of the differential equation. Magn Reson Med. 2001;45(2):299-310. doi:10.1002/15222594(200102)45:2<299::AID-MRM1039>3.0.CO;2-0

42. Muthupillai R, Clinic M. Spectrally-Selective Gradient Waveforms: Applications in MR Elastography. In: Proceedings of the 6th Annual Meeting of ISMRM, Sydney, Australia, 1998 (Abstract 2180)

43. Manduca A, Lake DS, Kugel JL, Rossman PJ, Ehman RL. Dispersion Measurements from Simultaneous Multi-Frequency MR Elastography. In: Proceedings of the $11^{\text {th }}$ Annual Meeting of ISMRM, Toronto, Ontario, Canada, 2003 (Abstract 550)

44. Riek K, Klatt D, Nuzha H, Mueller S, Neumann U, Sack I, Braun J. Wide-range dynamic magnetic resonance elastography. J Biomech. 2011;44(7):1380-1386. doi:10.1016/j.jbiomech.2010.12.031

45. Reiter R, Freise C, Jöhrens K, Kamphues C, Seehofer D, Stockmann M, Somasundaram R, Asbach P, Braun J, Samani A, Sack I. Wideband MRE and static mechanical indentation of human liver specimen: Sensitivity of viscoelastic constants to the alteration of tissue structure in hepatic fibrosis. J Biomech. 2014;47(7):1665-1674. doi:10.1016/j.jbiomech.2014.02.034

46. Maderwald S, Stock F, Galban CJ, Jeyrani R, Uffmann K, Liffers A, Quick HH, Ladd ME. High-Resolution 3D MR Elastography of Human Skin. In: Proceedings of the $13^{\text {th }}$ Annual Meeting of ISMRM, Miami, Florida, USA, 2005 (Abstract 2559) 
47. Lopez O, Amrami KK, Manduca A, Ehman RL. Characterization of the dynamic shear properties of hyaline cartilage using high-frequency dynamic MR elastography. Magn Reson Med. 2008;59(2):356-364. doi:10.1002/mrm.21474

48. Nemeth A, Marco L, Boutitie F, Sdika M, Grenier D, Rabilloud M, Beuf O, Pialat J. Reproducibility of in vivo magnetic resonance imaging $\mathrm{T} 1$ rho and $\mathrm{T}_{2}$ relaxation time measurements of hip cartilage at 3.0T in healthy volunteers. J Magn Reson Imaging. 2018;47(4):1022-1033. doi:10.1002/jmri.25799

49. Mariappan YK, Glaser KJ, Manduca A, Romano AJ, Venkatesh SK, Yin M, Ehman RL. High-frequency mode conversion technique for stiff lesion detection with magnetic resonance elastography (MRE). Magn Reson Med. 2009;62(6):1457-1465. doi:10.1002/mrm.22091 


\section{TABLES}

\begin{tabular}{|c|c|c|c|c|c|c|c|}
\hline \#Acquisition & $\begin{array}{c}\text { Number of } \\
\text { phase offsets }\end{array}$ & $\begin{array}{c}\text { Acquisition } \\
\text { time }\end{array}$ & $\mathrm{f}_{\text {exc }}(\mathrm{Hz})$ & $\mathrm{A}_{\text {exc }}(\mathrm{V})$ & $\mathrm{f}_{\mathrm{MEG}}(\mathrm{Hz})$ & $\mathrm{N}_{\mathrm{G}}$ & MEG shape \\
\hline 1 & 4 & $2 \min 8 \mathrm{~s}$ & 300 & 1 & 300 & 1 & trapezoidal \\
\hline 2 & 4 & $2 \min 8 \mathrm{~s}$ & 900 & 1 & 900 & 3 & trapezoidal \\
\hline 3 & 4 & $2 \min 8 \mathrm{~s}$ & 900 & 3 & 300 & 1 & trapezoidal \\
\hline 4 & 4 & $2 \min 8 \mathrm{~s}$ & 900 & 3 & 300 & 1 & sinusoidal \\
\hline 5 & 5 & $2 \min 40 \mathrm{~s}$ & $300: 900$ & $1: 3$ & 300 & 1 & trapezoidal \\
\hline
\end{tabular}

Table 1. Experimental MRE parameters 


\section{FIGURES}

a)

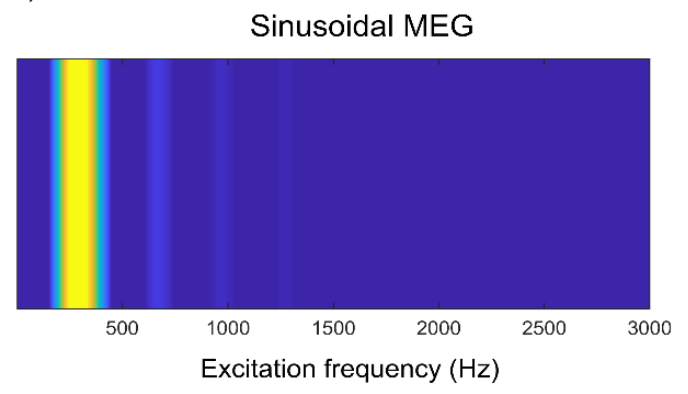

b)

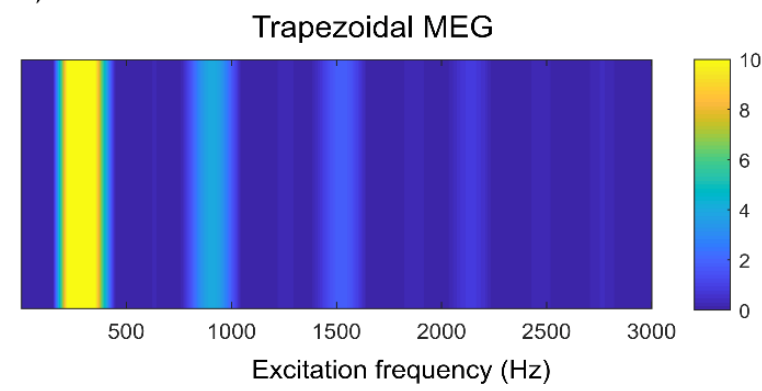

Figure 1. Accumulated phase with one cycle of $300 \mathrm{~Hz}$ MEG for various excitation frequencies and with different oscillating gradient shapes. A, Sinusoidal shaped; B, trapezoidal shaped. Values are in arbitrary units and were scaled from 0 to 10 for a better visualization. The periodical behavior of the sensitivity bands is visible when using a trapezoidal MEG. 

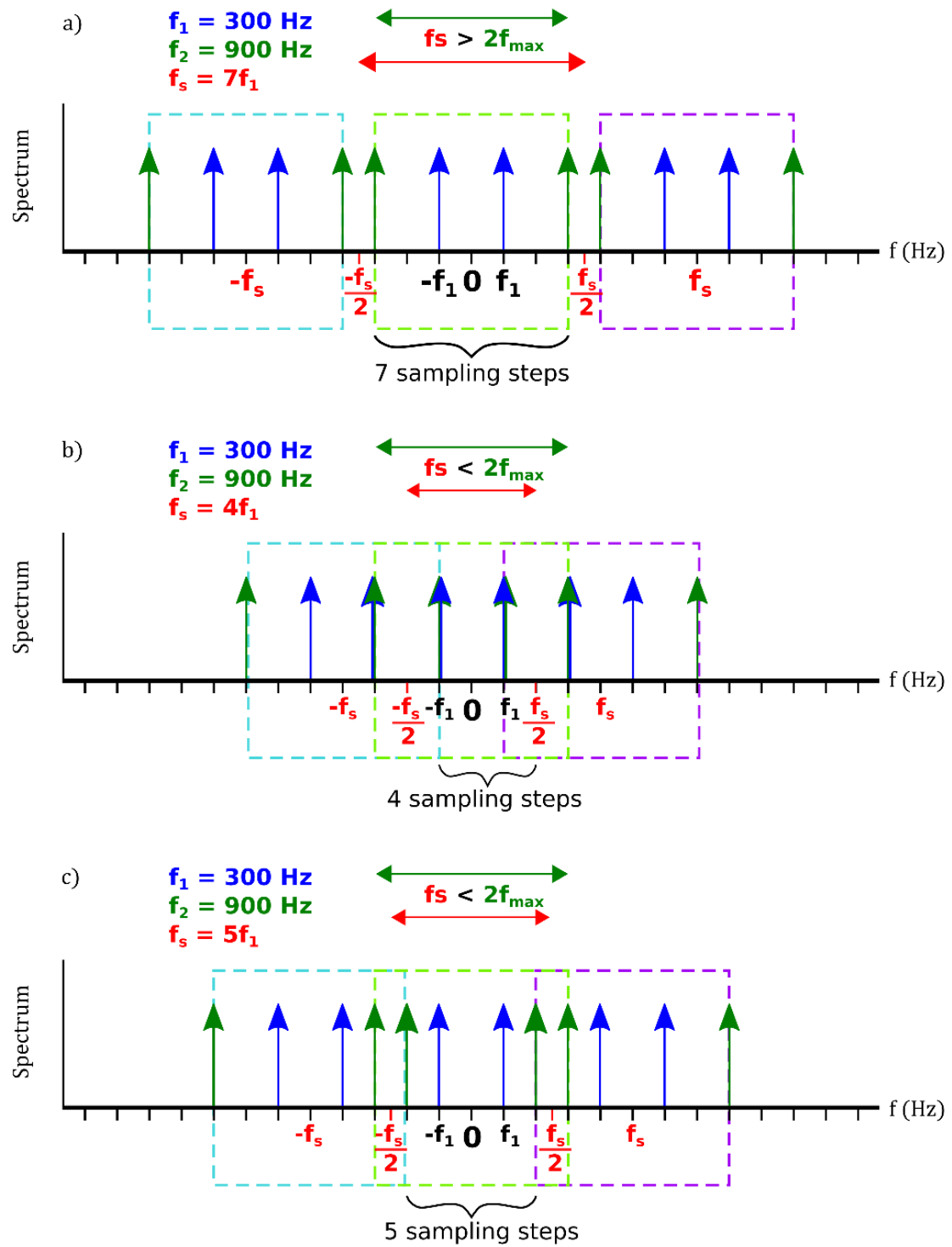

Figure 2. Different representations of the spectra obtained in the frequency domain after temporal Fourier transform of sampled points at a sampling frequency $f_{s}$. The original signal was a dual-frequency wave $\left(f_{1}, f_{2}\right)$ equal to $(300,900)$ Hz. A, The spectra when $f_{s}=$ $7 \mathrm{f}$, respecting the Shannon theorem. B, C, The spectra when a sampling not respecting the Shannon theorem is applied, producing a temporal aliasing. In B with $f_{s}=4 f_{1}$ the temporal aliasing produces an overlap of the two frequency components at the same acquired points. In $\mathrm{C}$ with $\mathrm{f}_{\mathrm{s}}=5 \mathrm{f}_{1}$ the temporal aliasing is controlled in order to obtain both frequency components at different acquired points, enabling separation of the two components. Green and blue arrows represent the Dirac delta functions of the 300 and $900 \mathrm{~Hz}$ components, respectively. 


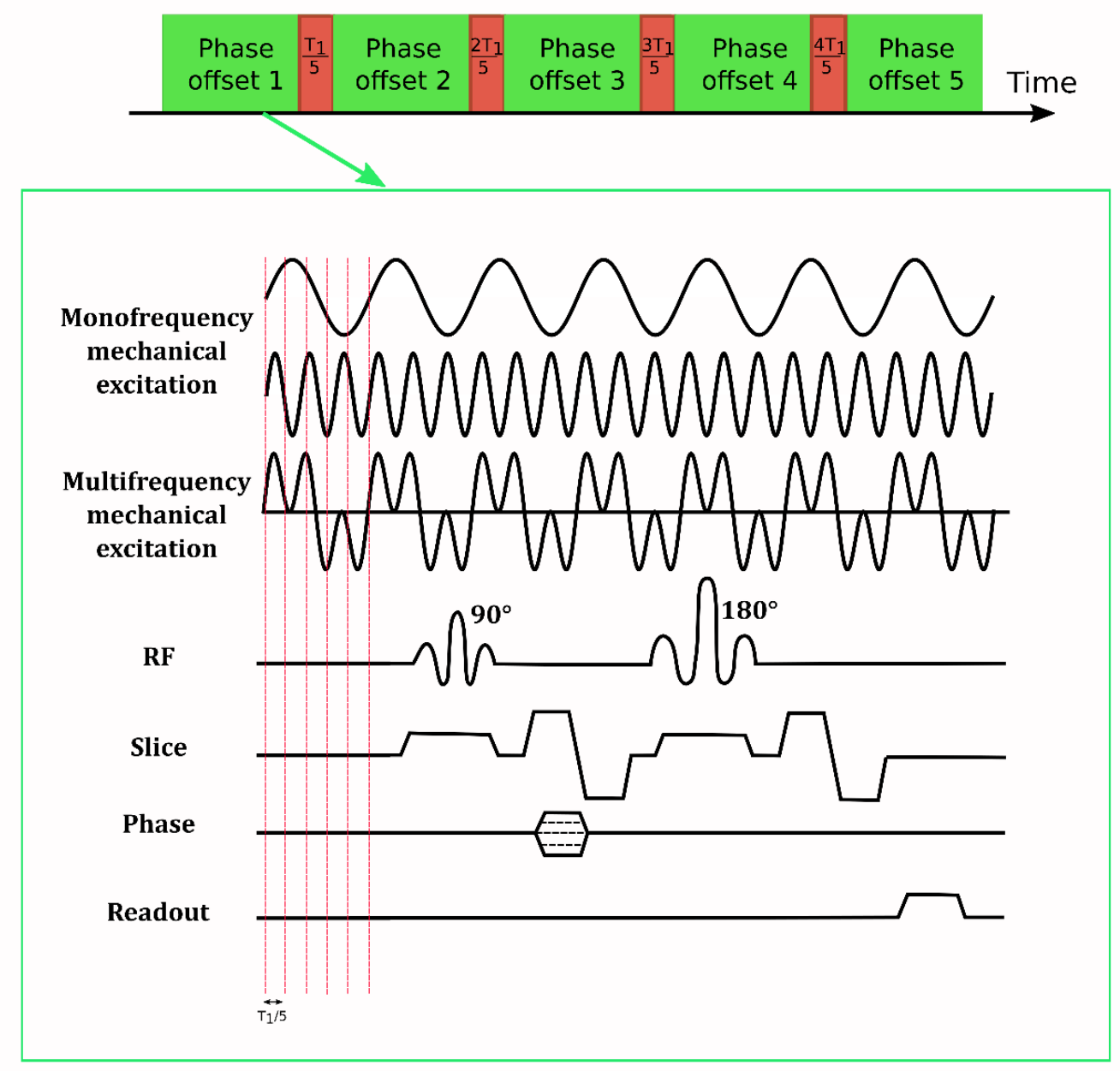

Figure 3. Chronogram of the multifrequency acquisition pulse sequence. The period of the lowest-frequency mechanical vibration is denoted as $\mathrm{T}_{1}$. After the complete image of a phase offset has been acquired (green square), a delay of $\mathrm{T}_{1} / 5$ (red square) is added to perform the acquisition for the next phase offset image. 

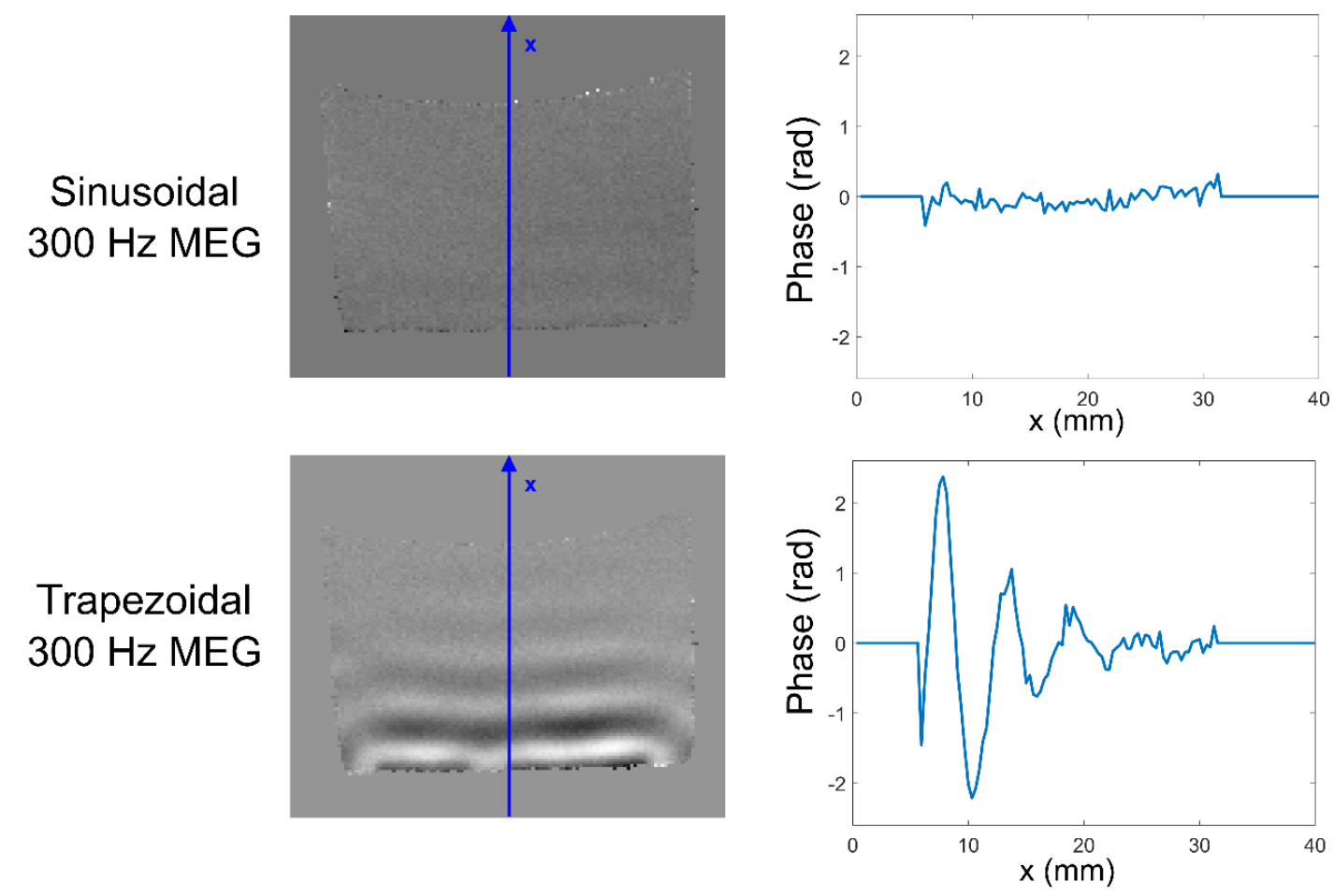

Figure 4. Wave propagation visualization on experimentally acquired phase images (left), and profiles following the blue arrow (right), when a $300 \mathrm{~Hz}$ MEG is applied (top, sinusoidal shaped; bottom, trapezoidal shaped) with a $900 \mathrm{~Hz}$ shear wave excitation, in radians. The motion is encoded when using a trapezoidal MEG. 


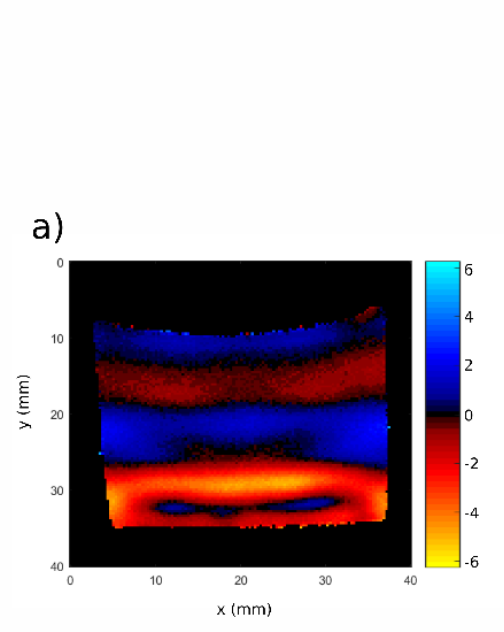

b)
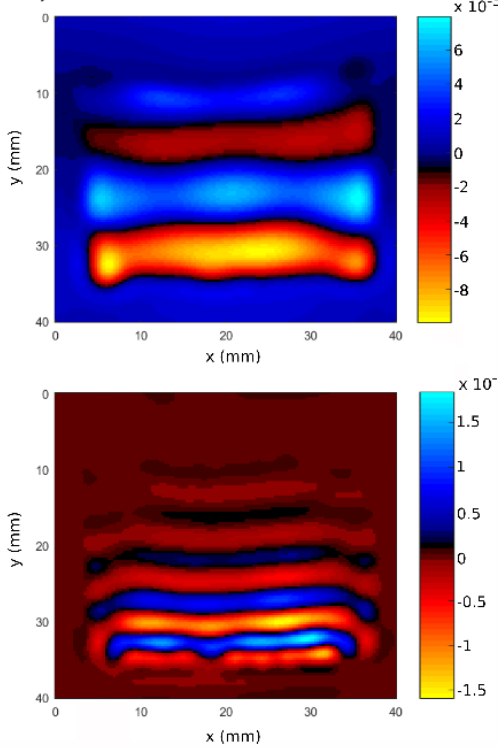

c)
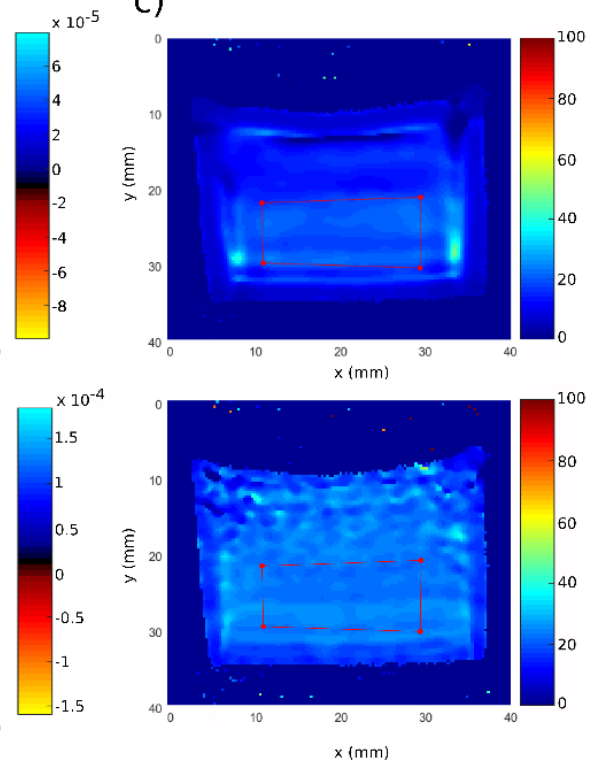

Figure 5. A, Phase image of the composite excitation (rad). B, Phase images (a.u.) and C, $\mathrm{G}^{\prime}$ elastograms $(\mathrm{kPa})$ of every extracted frequency component: top, $300 \mathrm{~Hz}$; bottom, 900 Hz. The ROI used for the computation of the shear storage modulus mean and standard deviations is shown with red lines on the maps in C. 


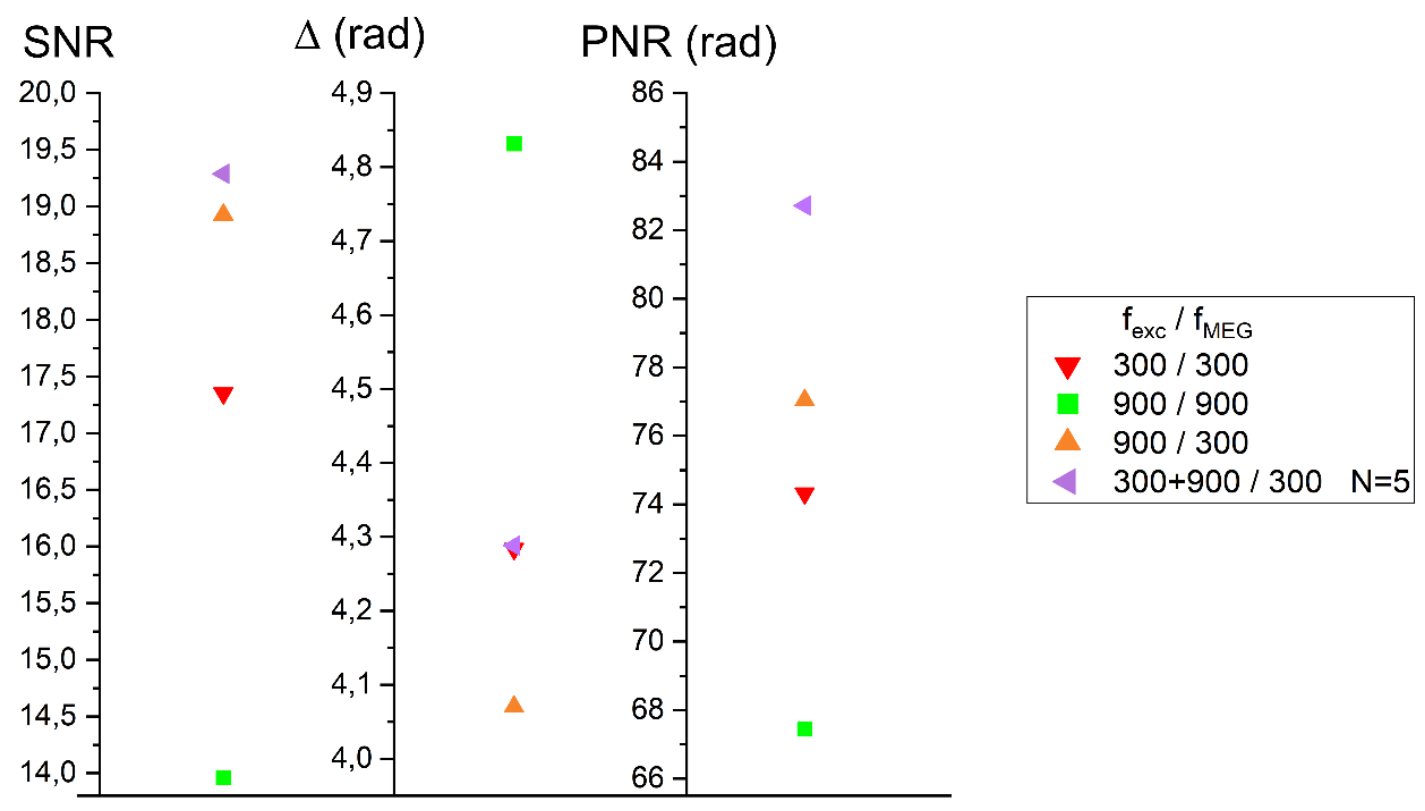

Figure 6. Plot of the SNR, $\Delta$ and PNR values obtained with the different acquisition modes. SNRs are in arbitrary units, $\Delta$ and PNRs in radians. Note that each parameter has its own vertical axis. Only the acquisitions with trapezoidal MEGs are considered here. In the legend: conventional monofrequency acquisition $(\boldsymbol{\nabla}, \mathbf{\square})$, harmonic monofrequency acquisition ( $\mathbf{\Delta})$ and dual-frequency acquisition $(\bullet)$. 


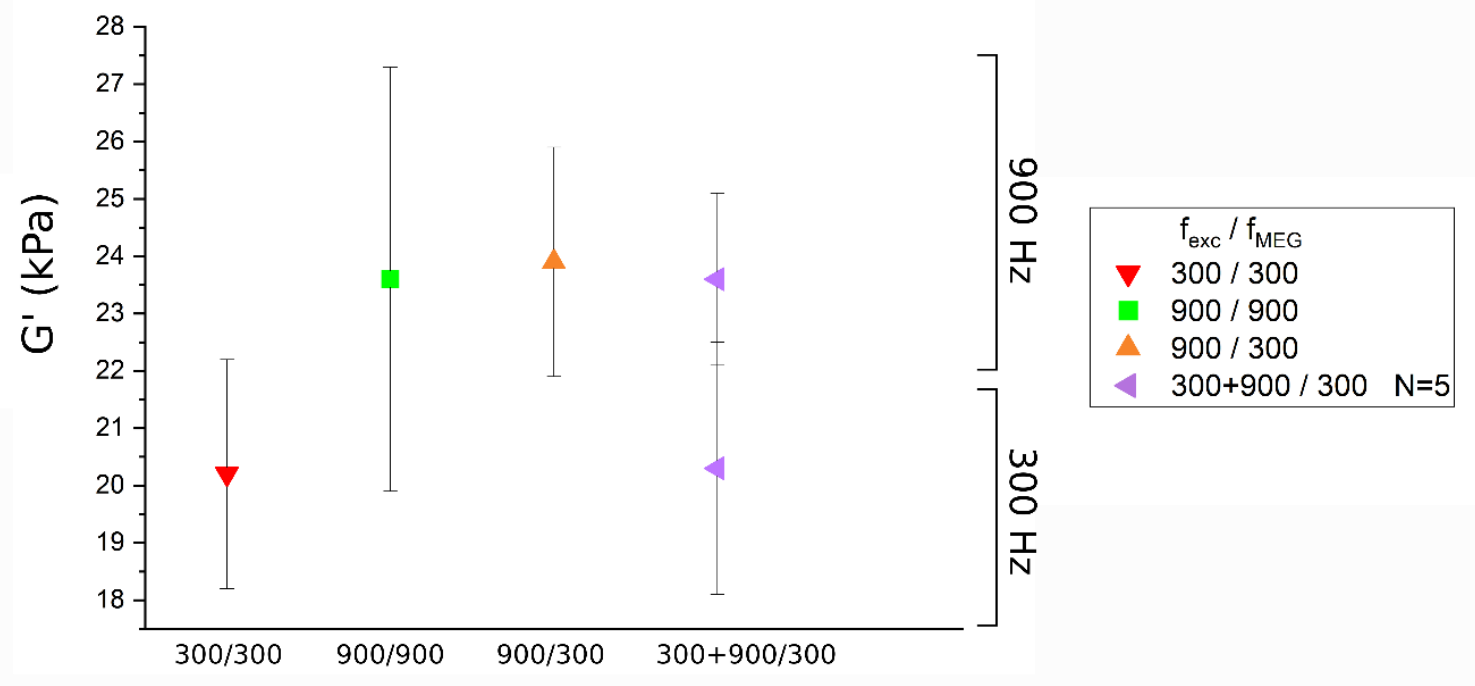

Figure 7. Mean values, with standard errors displayed as error bars, of the reconstructed shear storage modulus G0 for both excitation frequencies (300 and $900 \mathrm{~Hz}$ ) obtained with the different acquisition modes. Only the acquisitions with trapezoidal MEGs are considered here. An excellent agreement of the G0 values is found for all cases. 